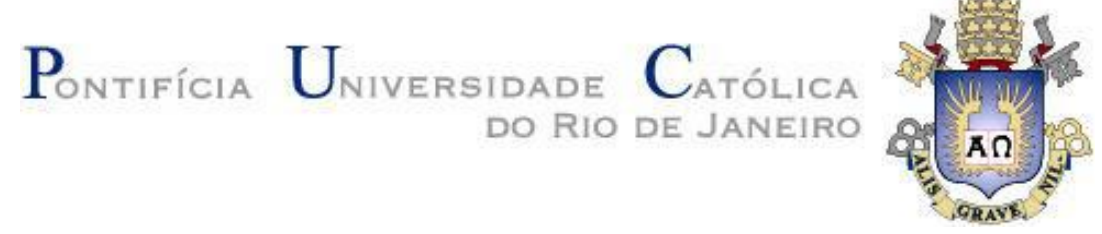

Wilson Cardoso Junior

\title{
A INTERCULTURALIDADE NO ENSINO DE ARTES VISUAIS DO COLÉGIO PEDRO II
}

Tese de Doutorado

Tese apresentada ao Programa de Pós-Graduação em Educação da PUC-Rio como requisito parcial para a obtenção do grau de Doutor em Educação.

Orientadora: Profa. Vera Maria Ferrão Candau 
Wilson Cardoso Junior

\section{A INTERCULTURALIDADE NO ENSINO DE ARTES VISUAIS DO COLÉGIO PEDRO II}

Tese apresentada como requisito parcial para obtenção do grau de Doutor pelo Programa de Pósgraduação em Educação do Departamento de Educação do Centro de Teologia e Ciências Humanas da PUC-Rio. Aprovada pela Comissão Examinadora abaixo assinada.

Prof á. Vera Maria Ferrão Candau

Orientadora

Departamento de Educação - PUC-Rio

Profa . Rosália Maria Duarte Departamento de Educação - PUC-Rio

Profa. Isabel Alice Oswald Monteiro Lelis

Departamento de Educação- PUC-Rio

Profo. Antonio Flávio Barbosa Moreira UCP/RJ

Profo. Aldo Victorio Filho Universidade do Estado do Rio de Janeiro

Prof ${ }^{\mathrm{a}}$ Monah Winograd Coordenadora Setorial do Centro de Teologia e Ciências Humanas PUC-Rio

Rio de Janeiro, 31 de Março de 2017. 
Todos os direitos reservados. É proibida a reprodução total ou parcial do trabalho sem autorização da universidade, do autor e da orientadora.

\section{Wilson Cardoso Junior}

Graduou-se em Licenciatura em Educação Artística/História da Arte pela Universidade do Estado do Rio de Janeiro (1984) e mestrado em Educação pela Pontifícia Universidade Católica do Rio de Janeiro (1999). Doutorando em Educação da Pontifícia Universidade Católica do Rio de Janeiro, com entrada em 2013. Professor assistente da Universidade Federal do Rio de Janeiro. Tem experiência na área de Educação, com ênfase em Ensino de Artes Visuais, atuando principalmente com os temas de ensino da arte, educação, interculturalidade e história da arte.

Ficha Catalográfica

Cardoso Junior, Wilson

A interculturalidade no ensino de artes visuais do Colégio Pedro II / Wilson Cardoso Junior; orientadora: Vera Maria Ferrão Candau. - 2017.

292 f: il.; $30 \mathrm{~cm}$

Tese (doutorado) - Pontifícia Universidade Católica do Rio de Janeiro, Departamento de Educação, 2017. Inclui bibliografia

1. Educação - Teses. 2. Interculturalidade crítica. 3. Educação intercultural. 4. Ensino de artes visuais. 5. Educação escolar. 6. Colégio Pedro II. I. Candau, Vera Maria Ferrão. II. Pontifícia Universidade Católica do Rio de Janeiro. Departamento de Educação. III. Título. 


\section{Agradecimentos}

Aos meus pais, in memorian, por tudo que carinhosamente semearam, principalmente o compromisso com o próximo, a educação, a justiça social e ao que a história ensina, especialmente sobre os povos da América do Sul.

À minha avó Josélia, minha tia Joselma e meu amigo-irmão Lavozier Formiga Casimiro, in memoriam, que sempre me ajudaram tanto e que se foram durante a realização desse trabalho.

À inesquecível Professora Noêmia Varela, in memoriam, uma das baluartes da Arte-Educação brasileira, que desde 1987 me incentivou a investir na pesquisa sobre o ensino de artes visuais no Brasil.

À minha irmã Janine pelo apoio imenso, carinho e escuta competente e paciente; a sua presença fraterna foi uma das bases afetivas fundamentais que possibilitaram esse trabalho e o iluminaram com o seu exemplo de integridade, dignidade e bom humor que foram imprescindíveis, principalmente quando a vida deixava tudo fora do lugar.

Ao meu filho Gregório por todo afeto, ajuda em traduções e compreensão com a ausência do pai durante essa jornada em que a vida familiar e social esteve praticamente "entre parênteses".

À Adriana, meu amor, pelo companheirismo, sabedoria, leituras, reflexões, solicitude e paciência inenarráveis; mas, sobretudo, pela imensa alegria de viver que me iluminou nos momentos mais difíceis dessa trajetória, me ajudando a ver "a parte cheia do copo" e, às vezes, pela simples promessa de "logo mais vamos ao cinema".

À Professora Vera Maria Ferrão Candau, que desde o mestrado, realizado na segunda metade dos anos 1990, tem me concedido o privilégio de contar com a sua sabedoria e orientação rigorosa, segura e profundamente respeitosa, me alertando com o seu conhecimento e assertividade para os possíveis tropeços e desvios de foco, assim como abriu portas e horizontes que inspiraram novas rotas e soluções para os desafios encontrados nesse estudo. 
À querida amiga Maria Thereza Jorand por ter contribuído de maneira fundamental para a recuperação de minhas memórias afetivas, reavaliação de processos pessoais e ressignificação de valores que deram início ao percurso interior para a realização desse trabalho.

À amiga Selma Wainstock, por tantas trocas de ideias, dicas e a escuta sensível e generosa com que pude contar; além de me ajudar com sessões de gargalhadas revitalizantes sobre as dificuldades surgidas nesse percurso.

À amiga Leila Gross que com enorme generosidade me acudiu nos momentos finais e fez a revisão do texto e contribuiu nas traduções necessárias ao trabalho.

Ao amigo Pablo das Oliveiras por em 2013, no começo dessa trajetória, ter tido uma escuta tão atenciosa às primeiras intenções desse trabalho e ter ajudado a construir seus propósitos finais.

À Greice Cohn, amiga de longa data de quem pude usufruir de tantos ensinamentos, compartilhamentos e disponibilidade; essa tese também é fruto de nossos entrecruzamentos profissionais (como professores e pesquisadores) e da possibilidade de olhar de perto para a sua busca constante por um ensino de Artes Visuais digno e capaz de se apropriar das urgências de nosso tempo.

À Fátima Ivone, parceira de Colégio Pedro II na Unidade Escolar Engenho Novo II, nos anos 1990, por me ajudar imensamente com a contextualização de um novo CP II que eu não conhecia.

À amiga Andrea Penteado pela afetividade, generosidade, coragem, sensibilidade e criticidade desafiadora que iluminaram esse estudo sob diversos ângulos; além de sempre me oferecer refúgio na divina Picinguaba.

À Silvia Soter pela amizade, incentivo, escuta sensível e cumplicidade tecida a partir das similaridades de nossas trajetórias profissionais e acadêmicas.

Aos professores e professoras de Artes Visuais do Colégio Pedro II que prestaram colaboração preciosa ao responderem os questionários e se disponibilizarem as entrevistas com enorme generosidade, assim como aos/as funcionários/as e professores/as do NUDOM (Núcleo de Documentação e Memória do CPII). 
Ao Colégio Pedro II por permitir a minha pesquisa e me acolher de forma atenciosa nos diferentes campi e setores em que fiz solicitações necessárias para a realização desse trabalho.

À Professora Sandra Barata Gomes, chefe do Departamento de Desenho e Artes Visuais, pela paciência e apoio incessante, disponibilizando acessos, informações e conhecimentos que foram de grande valia para a realização desse trabalho.

Aos/Às Professores/a Antônio Flávio Moreira, Isabel Alice Lelis, Rosália Duarte, Aldo Victório Filho, Adélia Maria Nehme Simão e Koff por aceitarem a incumbência de avaliar, compor a banca final e contribuir para a conclusão desse estudo.

Ao Professor Marcelo Andrade, amigo desde a época de mestrado nos anos 1990, professor exuberante que integrou a banca final (como suplente) e participou das bancas dos Exames de Qualificação, tendo contribuído com leituras perspicazes que me ajudaram desde a formulação inicial do projeto de pesquisa até a sua versão final.

Às colegas do GECEC - Grupo de Estudos do Cotidiano Escolar e Culturas pela convivência e trocas que abriram portas e horizontes e aguçaram minha percepção sobre a temática da Educação Intercultural. Foram elas - Adélia Maria Nehme Simão e Koff , Daniela Valentim, Yrama, Susy Sacavino, Natalia Tavares Rios, Dila Carvalho, Angela Sousa, Ana Paula Soares, Ana Paula Santos, Clea Ferreira, Cristiane Taveira e Guilherme de Alcântara.

Aos/Às servidores/as técnicos/as da Secretaria da Pós-Graduação em Educação da PUC-Rio, pelo auxílio, atenção e estrutura, especialmente à Nancy Ferreira, Marnie Siqueira de Oliveira e Geneci Félix.

À Faculdade de Educação da Universidade Federal do Rio de Janeiro, especialmente aos/às colegas do Departamento de Didática, pela licença para estudos que foi fundamental para a realização desse trabalho.

Agradeço a Padmasambhava e São Jorge por todas as bênçãos, saúde e proteção ao longo desse caminho. 


\section{Resumo}

Cardoso Junior, Wilson; Candau, Vera Maria Ferrão, A interculturalidade no ensino de Artes Visuais do Colégio Pedro II. Rio de Janeiro, 2017. 292p. Tese de Doutorado - Departamento de Educação, Pontifícia Universidade Católica do Rio de Janeiro.

O presente trabalho teve por objetivo conhecer concepções e práticas voltadas para a promoção do ensino intercultural de Artes Visuais na educação escolar. Ele partiu do entendimento da ancoragem histórico-social da ideia de arte hegemônica que tem produzido a inexistência das artes dos povos subalternizados historicamente por processos de outrificação e periferização que as confina ao passado da história da arte. O referencial teórico articula os conceitos de interculturalidade crítica (WALSH, 2009, 2011), a concepção de educação intercultural crítica (CANDAU, 2006, 2009a, 2009b, 2014, 2016) e a ecologia de saberes (SANTOS, 2006, 2008, 2010) em interlocução com as concepções de ensino de Artes Visuais pós-modernista (BARBOSA, 1981, 1998, 2002, 2005), ensino intercultural de artes (RICHTER, 2002, 2003, 2008; MASON, 2000), currículo integrado e disciplina "mal estruturada" (PARSONS, 2005) e arte pósautônoma (CANCLINI, 2012). Foi realizado um estudo de caso sobre a interculturalidade e o ensino de Artes Visuais no Colégio Pedro II utilizando principalmente entrevistas semi-estruturadas. Os resultados apontam para a existência de um ensino de artes "out of the box", baseado nos conceitos de "arte como experiência" e "arte em campo expandido", que vem investindo na interculturalidade como princípio epistemológico a partir da conjugação das práticas de atelier com histórias de vida dos/as alunos/as; do currículo por eixos temáticos contaminado pela arte contemporânea e a arte popular, com ênfase nas artes indígenas, africanas e afro-brasileira; da formação docente continuada em serviço com foco nas noções de professor/a-pesquisador/a e professor/a-curador/a; do ensino de Artes Visuais antirracista articulado com a identidade cultural de seus/suas praticantes. Essa perspectiva articula-se com as demandas por justiça cognitiva para uma educação digna e tem colocado em questão o conceito de qualidade do ensino de Artes Visuais presidido pela história da arte de raiz única linear e eurocêntrica.

\section{Palavras-chave}

Interculturalidade crítica; educação intercultural; ensino de Artes Visuais; educação escolar; Colégio Pedro II. 


\section{Abstract}

Cardoso Junior, Wilson; Candau, Vera Maria Ferrão, (Advisor) Interculturality in Visual Arts teaching at Colégio Pedro II, Rio de Janeiro, 2017, 292p. Tese de Doutorado - Departamento de Educação, Pontifícia Universidade Católica do Rio de Janeiro.

The present paper had as main objective to gather practices and conceptions aimed at the promotion of intercultural teaching of Visual Arts in school education. Based on the understanding that the social-historical idea of hegemonic art has produced the non-existence of the arts among the subordinated people by processes of 'outrification" and 'peripherization' (exclusion to periphery) restricting them in the past of the history of the art. The theoretical reference articulates the concepts of critical interculturality (WALSH, 2009, 2011), the conception of intercultural critical education (CANDAU, 2006, 2009a, 2009b, 2014, 2016) and the ecology of knowledge (SANTOS, 2006, 2008, 2010) in connection with the conceptions of post-modernism visual arts teaching (BARBOSA, 1981, 1998, 2002, 2005), intercultural teaching of arts (RICHTER, 2002, 2003，2008; MASON, 2000) integrated curriculum and poorly "structured disciplines" (PARSONS, 2005) and post-autonomous art (CANCLINI, 2012). A case study of the interculturality and Visual Arts at Colégio Pedro II was undertaken, mainly using semi-structured interviews. The results point out the existence of an 'out-of-the-box' arts teaching based upon the concepts of "arts" as experience and 'art in large field' which has invested in interculturality as the epistemological source of workshops where students tell their life stories; of the curriculum by thematic main points touched by contemporaneous art and pop art, with emphasis on native, African and AfroBrazilian art; of ongoing teacher training with focus on the meaning of the relationship between teacher/researcher and teacher/curator; of antiracist visual arts teaching connected to the cultural identities of the practitioners. This perspective is in articulation with the demands for cognitive justice and a dignified education and has put forward as a critical issue the concept of quality of visual arts teaching controlled by the single root history of art - linear and Eurocentric.

\section{Keywords}

Critical Interculturality; intercultural education; Visual Arts teaching; school education; Colégio Pedro II. 


\section{Sumário}

$\begin{array}{lll}1 & \text { Introdução } & 18\end{array}$

2 Referencial teórico-metodológico 29

$\begin{array}{lll}2.1 & \text { Eixos teóricos } & 29\end{array}$

$\begin{array}{lll}2.1 .1 & \text { Interculturalidade crítica }\end{array}$

2.1.2 interculturalidade Crítica 37

2.1.3 Educação intercultural crítica 41

2.1.4 Ensino de artes contemporâneo e a interculturalidade 45

2.2 Abordagem metodológica 57

2.2.1 O campo de pesquisa 57

2.2.2 Instrumentos de pesquisa 60

2.2.2.1 Questionários 61

2.2.2.2 Entrevistas 61

2.2.2.3 Análise documental 69

2.2.2.4. Observação 70

$\begin{array}{lll}2.3 & \text { Análise de dados } & 72\end{array}$

O Colégio Pedro II - Contexto atual, tradições educativas e

3 ensino de Artes Visuais 73

O Colégio Pedro II "mudando de pele" ": mudanças e manutenções com a nova identidade jurídica institucional

3.1 (Instituição Federal de Ensino - IFE) 73

"A ilusão a ser evitada"': a tradição assimilacionista, o fim da

3.2 jubilação e a adoção da educação inclusiva 81

"O silêncio é embranquecedor" ": o Núcleo de Estudos Afrobrasileiros, o currículo eurocentrado do CP II e as Leis №

$3.310639 / 2003$ e № 11645/2008

As diferenças na sala de aula: a percepção de docentes de

3.4 Artes Visuais

"Eles tinham questões da escola, principalmente da relação

3.5 discentes

3.6 O ensino de Artes Visuais no Colégio Pedro II 113

3.6.1 Breve histórico institucional da disciplina Artes Visuais 116

3.6.2 história, currículo e disputas no DDAV 
4 A corrosão do eurocentrismo 129

4.1 A história da arte como paradigma curricular 131

Eurocentrismo, colonização cultural e produção de

4.2 inexistências no ensino de Artes Visuais 139

4.3 Visões sobre as Leis 10.639/2003 e 11.645/2008 151

Reformulação curricular, formação inicial e renovação do

4.4 quadro docente no DDAV 159

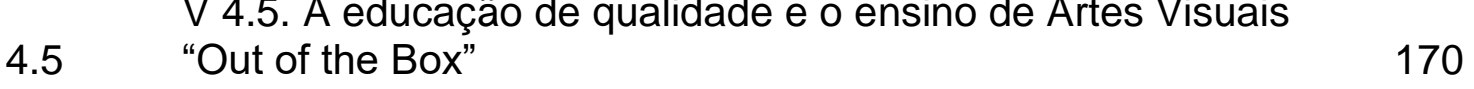

Concepções e práticas interculturais no ensino de Artes

$5 \quad$ Visuais do Colégio Pedro II

Os sentidos da intercuturalidade segundo os/as

5.1 professores/as de Artes Visuais do CPII

Caminhos da interculturalidade no Colégio Pedro II - o

5.2 ensino de Artes Visuais do "Pedrinho"

A conjugação de práticas de atelier com histórias de vida

O olhar multidisciplinar e o conceito de arte em campo

5.2.2 expandido

O currículo por eixos temáticos e os conhecimentos/saberes

5.2.3 para a interculturalização do ensino de Artes Visuais

5.2.4 Formação continuada em serviço, pesquisa e curadoria 217 O Ensino de Artes Visuais antirracista, empoderamento e
identidade cultural

5.3 O projeto "Cultura Afro-brasileira - uma integração possível" 238

6 Considerações Finais 249

7 Referências bibliográficas 263

$8 \quad$ Anexos 


\section{Lista de figuras}

Figura 1 - Tinta amarelo-pele 18

Figura 2 - Lápis cor de pele 18

Mapa conceitual da ecologia de saberes na perspectiva

Figura 3 - da interculturalidade crítica 42

Figura 4 - Aluna do 9o ano pintando - Campus Centro 95

Figura 5 - Pintura de aluna do 9 ano - Campus Centro 95

Figura 6 - Colagem de aluno do 9a ano - Campus Centro 95

Figura 7 - Colagem de aluno do 9ำ ano - Campus Centro 95

Figura 8 - Logotipo do CP II 103

Figura 9 - Logotipo do Feminismo de 3/4 103

Figura 10 - Logotipo do NEAB-CP II 103

Figura 11 - Logotipo do Retrato Colorido 103

Figura 12 - Notícia sobre o Saiato de aluno/as do CP II 104 Foto de capa do facebook do coletivo Retrato Colorido

Figura 13 - - 2016

Foto do período de ocupação do Campus Niterói -

Figura 14 - 2016

106

Foto do movimento de alunas do CP II

Figura 15 - "NãoMerecemosSerEstupradas" -2016

Foto do movimento de alunas/os do CP II "Todos por todas"

Figura 16 - -2016

107

Figura 17 - Postagem no facebook do Coletivo Feminismo 3/4 108

Foto de cartaz de alunos/as colado na sala do

Figura 18 - Departamento de Sociologia

Figura 19 - Vista do início para o final do mural do $\mathrm{CH} \mathrm{II}$

Figura 20 - Vista do final para o início do mural do $\mathrm{CH}$ II 135 representações de figuras fenotipicamente não-

Figura 21 - brancas no mural do $\mathrm{CH}$ II

Figura 22 - Exposição Bordaduras Contemporâneas no Espaço Cultural

Figura 23 - Exposição Bordaduras Contemporâneas no Espaço Cultural

Figura 24 - fotografia do século XIX"

Figura 25 - Cristóvão I

Figura 26 - Caixa de lápis de cor UNIAFRO 
Figura 27 - Trabalho - "Achei a minha cor" 228

Figura 28 - Trabalho - "Achei a minha cor" 228

Figura 29 - Trabalho - "Achei a minha cor" 228

Figura 30 - Trabalho - "Achei a minha cor" 228

Reprodução do desenho do Engenho do Buriti, feito por Hércules Florence, durante a Expedição Langsdorff, no século XIX, exposto na Sala da

Figura 31 - - Daniel Pellegrim Sanchez

Figura 32 - Árvore da vida e Árvore genealógica 240

Figura 33 - Autorretratos de estudantes do $\mathrm{CH} \mathrm{I} 240$

Figura 34 - Rubem Valentim 240

Figura 35 - Obra de Rubem Valentim 240

Figura 36 - "Objetos de poder" 240

Figura 37 - Atividade de pesquisa em sala de aula 241

Figura 38 - Produção coletiva de estudantes 241

Figura 39 - Publicações trabalhadas em sala de aula 241

Figura 40 - Entrada do CQS 241

Figura 41 - Via de acesso ao CQS 241

Figura 42 - Roda de capoeira na CQS 242

Figura 43 - Palestra de Luiz Sacopã na CQS 242

Depoimentos e desenhos em tecido sobre a visita à

Figura 44 - CQS

Figura 45 - Depoimento de aluna sobre a visita à CQS 242

Visita de alunos/as do Campus Tijuca II à Comunidade

Figura 46 - Caiçara da Praia do Sono

Visita de alunos/as do Campus Tijuca II à Comunidade

Figura 47 - Caiçara da Praia do Sono 


\section{Lista de quadros}

Levantamento de Estudos sobre Interculturalidade e

Quadro 1 - Ensino de Artes Visuais

Experiência docente no Colégio Pedro II dos/as

docentes de Artes Visuais entrevistados/as (tempo de

Quadro 2 - $\quad$ serviço e lotação na educação básica)

Quadro 3 - Campi da Educação Básica do Colégio Pedro II / 2016

Quadro 4 - (Dados de 2014)

Cor / Raça dos/as Estudantes do Ensino Básico do

Quadro 5 - $\quad$ Colégio Pedro II / 2014

Levantamento de Pesquisas sobre Ensino da Arte no

Quadro 6 - CP II / Organizadas por ano de conclusão

SÍNTESE DO CURRÍCULO DE ARTES VISUAIS DO

Quadro 7 - EF 1 - CP II / 2015

SÍNTESE DO CURRÍCULO DE ARTES VISUAIS DO

Quadro 8 - EF 2 - CP II /2015

SÍNTESE DO CURRÍCULO DE ARTES VISUAIS DO

Quadro 9 - 10 ANO EMR - CP II /2015

Formação Inicial dos/as Docentes Efetivos do CP II por

Quadro 10 - Ano de Ingresso (Dados de 2015)

Formação em Pós Graduação dos Docentes de Artes

Quadro 11 - Visuais do Colégio Pedro II (Dados de 2015) 


\section{Lista de abreviaturas}

A - Antigo/a

Am - Amarela/o (cor/raça)

$\mathrm{Br}$ - Branca/o (cor/raça)

ANPAP - Associação Nacional de Pesquisadores em Artes Plásticas

ANPEd - Associação Nacional de Pós-Graduação e Pesquisa em Educação

ADCPII - Associação de Docentes do Colégio Pedro II

CINEAB - Cine-Núcleo de Estudos Afro-Brasileiros

CNPq/MCTI - Conselho Nacional de Desenvolvimento Científico e Tecnológico

CONSUP - Conselho Superior

CONEPE - Conselho de Ensino, Pesquisa e Extensão

CP II - Colégio Pedro II

CH I - Campus Humaitá I

CH II - Campus Humaitá II

CQS - Comunidade Quilombola Sacopã

CSC I - Campus São Cristóvão I

CSC II - Campus São Cristóvão II

CSC III - Campus São Cristóvão III

CT I - Campus Tijuca I

CT II - Campus Tijuca II

DBAE - Discipline Based Art Education

DDAV - Departamento de Desenho e Artes Visuais

DDEA - Departamento de Desenho e Educação Artística

DP - Departamento Pedagógico

DPSEF - Departamento do Primeiro Segmento do Ensino Fundamental

EC - Espaço Cultural

EF1 - Ensino Fundamental / Anos Iniciais

EF2 - Ensino Fundamental / Anos Finais

EI - Educação Infantil

ELOS - Núcleo de Estudos e Ações em Gênero e Sexualidade

EM - Ensino Médio

EMI - Ensino Médio Integrado 
EMI-PROEJA - Ensino Médio Integrado à Educação Profissional na modalidade de Educação de Jovens e Adultos

EMR - Ensino Médio Regular

EPCT - Rede Federal de Educação Profissional, Científica e Tecnológica

ERER - Educação das Relações Étnico-Raciais

ES - Ecologia de Saberes

e-SIC - Sistema de Acesso à Informação do Governo Federal

FAEB - Federação de Arte Educadores do Brasil

FCT - Fórum de Comunidades Tradicionais de Angra dos Reis, Paraty e Ubatuba

FN - Frente Negra

GECEC - Grupo de Estudos Cotidiano Escolar e Cultura/s

GEPARREI - Grupo de Estudos, Pesquisas e Ações sobre Racismo e Relações

Étnicorraciais e Indígenas

I - Intermediário

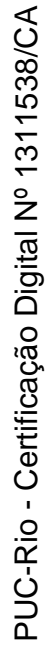

IBC - Instituto Benjamin Constant

IBGE - Instituto Brasileiro de Geografia e Estatística

IFE - Instituição Federal de Ensino

In - Indígena (cor/raça)

Inep - Instituto Nacional de Pesquisas em Educação

Ipea - Instituto de Pesquisa Econômica Aplicada

LDBEN - Lei de Diretrizes e Bases da Educação Nacional

MA - Muito/a Antigo/a

MEC - Ministério de Educação

MV - Mural da vergonha

NAPNES - Núcleo de Apoio às Pessoas com Necessidades Educacionais

Especiais

NEAB - Núcleo de Estudos Afro-Brasileiros

NEAB-CP II - Núcleo de Estudos Afro-Brasileiros do Colégio Pedro II

NEABI - Núcleo de Estudos Afro-brasileiros e Indígenas

NEPAG - Núcleo de Estudos e Pesquisa audiovisuais em Geografia

NUDOM - Núcleo de Documentação e Memória do Colégio Pedro II

NUTH - Núcleo Transdisciplinar de Humanidades

OTSS - Observatório de Territórios Sustentáveis e Saudáveis da Bocaina

PA - Pardo/a (cor/raça) 
PCNs - Parâmetros Curriculares Nacionais

PVF - Prova de Verificação Final

PG - Pós-Graduação

PGE - Programa Geral de Ensino do Colégio Pedro II

PI - Prova Institucional

PPP - Projeto Político Pedagógico

PPPI - Projeto Político Pedagógico Institucional

Pr - Preto/a (cor/raça)

PRD - Programa de Residência Docente

PROEN - Pró-Reitoria de Ensino

PROAd - Pró-Reitoria de Administração

PRODI - Pró-Reitoria de Planejamento e Desenvolvimento Institucional

PROEJA - Programa de Educação de Jovens Adultos

PROGESP - Pró-Reitoria de Gestão de Pessoas

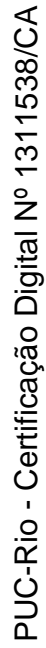

PROPGPEC - Pró -Reitoria de Pós-Graduação, Pesquisa, Extensão e Cultura

SEB/MEC - Secretaria de Educação Básica / Ministério da Educação

SECADI/MEC - Secretaria de Educação Continuada, Alfabetização e

Diversidade/Ministério da Educação

SRM - Sala de Recursos Multifuncionais do Tipo B

SESOP - Seção de Supervisão e Orientação Pedagógica

SIAAC - Sistema de Assentamento Acadêmico

SINDISCOPE - Sindicato dos Servidores do Colégio Pedro II

TCU - Tribunal de Contas da União

UFF - Universidade Federal Fluminense

UFRJ - Universidade Federal do Rio de Janeiro

UERJ - Universidade Estadual do Rio de Janeiro 
Ocupar é apoderar-se do espaço, permitindo que ele se torne o que somos. É o sinal físico da impossibilidade de se limitar idéias Para além da reivindicação de um espaço, ocupar é também um discurso simbólico corporal. É apenas através dos corpos ocupantes que criamos redes de solidariedade, aproximando as pessoas no âmbito físico e permitindo que se reconheçam nas opressões que sofrem. Ocupar é um grito de NÃO a esse governo indialogável. Não ao congelamento de gastos, não a liberdade de pensamento, não ao retrocesso das políticas sociais ocupar é dizer em união que incomodaremos MUITO os filhos de empresários que se recusam a estudar conosco porque não sairemos das universidades, não sairemos das escolas não abdicaremos dos nossos sonhos por uma minoria que pode pagar pelo país Assim encheremos as escolas e as ruas de pretos e pobres Ainda podemos ouvir correntes que nossos antepassados usaram, e não aceitaremos estar sob essa condição novamente. Ocupemos esse lugar que sempre foi nosso. Ocupemos porque não aceitaremos nada menos do que isso.

(Trecho de texto declamado com muita emoção por uma aluna durante a Assembléia da Ocupação do o Campus Realengo II do Colégio Pedro II, do dia 20 de outubro. Disponível em - https //www.facebook.com/ocupaCP2realOCUPA CPII REAL postagem feita em 22/11/2016 - acesso em 09/01/2017) 